\title{
Damage assessment in wind turbine technology
}

\author{
Wiesław Ostachowicz ${ }^{1,2^{*}}$, Pawet H. Malinowski ${ }^{1}$, Rohan Soman $^{1}$ and Tomasz Wandowski ${ }^{1}$ \\ ${ }^{1}$ Institute of Fluid-Flow Machinery, Polish Academy of Sciences, 80-231, Gdansk, Poland \\ ${ }^{2}$ Warsaw University of Technology, Faculty of Automotive and Construction Machinery \\ Engineering, 02-524, Warsaw, Poland
}

\begin{abstract}
The economic efficiency of wind turbines WT is strictly dependent on their availability and reliability. This problem is most important in the case of offshore wind turbines OWT. There is a search for new materials, new manufacturing process, and new rules of structural designs. The aim of it, is to achieve far tighter safety margins than previously developed wind turbine solutions. Attention of many researchers is focused on the problem of WT/OWT reliability.

In this paper Structural Health Monitoring (SHM) approach for wind turbines is presented. This approach is based on such topics as piezoelectric transducers, elastic waves propagation phenomenon, fibre Bragg gratings sensors, structural vibrations analysis. The combination of mentioned techniques allows to perform efficient both local and global SHM of the OWT.

Research results presented in this paper are mainly related to fibre reinforced polymer materials FRPs due to fact that they are widely utilized in wind turbine blades manufacturing. However, certain results for metallic structures (tower structure) were also presented.

Considered above investigations are intended to develop future SHM techniques that include sensors, hardware, software and methodologies of WT/OWT structural assessment.
\end{abstract}

\section{Introduction}

Wind Energy is seen as one of the most promising solutions to man's ever increasing demands of a clean source of energy. The use of wind energy has received an impetus due to the advancements in the field of materials engineering. Newer, bigger wind turbines are now possible which are more robust, and lighter in weight. The main drawback of the wind energy is the high initial costs for setting up and high maintenance costs. These high initial cost make the energy more expensive than the conventional energy sources like fossil fuels and nuclear and hence has not been widely accepted. In order to reduce the cost of generation, there is effort to increase the life-time of the wind turbines, reduce maintenance costs and ensure high availability [1].

The lifetime may be increased by ensuring a more robust design while the maintenance cost may be lowered and the high availability ensured through the use of condition monitoring (CM) and structural health monitoring (SHM). SHM allows early detection of damage and

\footnotetext{
* Corresponding author: wieslaw@imp.gda.pl
} 
allows maintenance planning which reduces the cost. Furthermore, it can allow us to avoid unnecessary downtime, hence increasing the availability of the system.

The wind turbine is a complex machine consisting of electrical and structural components. The structural components include the gear box, tower, rotor and several auxiliary mechanisms like the yaw system, pitch system etc. Considerable amount of research has been carried out in the area of SHM of rotating machinery which specifically deals with the CM of the gearbox system and can be found in $[2,3]$. The auxiliary systems although important will not cause catastrophic failure of the system and hence are not significant. The SHM of the structural components on the other hand is essential and a big challenge due to the specific problems associated with them. The study in this area can be classified as the one dedicated to the metallic structures including the support structure and the composite parts including the rotor blades. Due to the very different operating conditions and the dominant failure modes of these systems different SHM techniques need to be used for the monitoring based on the advantages and disadvantages of the individual techniques.

\section{Metallic Structures}

As can be seen in Fig. 1, the support \& housing sub-assembly of the wind turbine has the highest downtime per failure. This is attributed to the need of specialized equipment needed for the maintenance or the replacement of the sub-assembly. The numbers indicated are for on-shore wind turbines and are expected to go even higher for off-shore structures. Thus the support structure foundation is one of the key components of the wind turbine that need to be closely monitored. The foundation of the wind turbine is exposed to the most extreme environmental conditions including, salinity, extreme temperatures, scouring, drifting sea ice and higher possibility of collision with passing vessels and as such has to be carefully monitored.

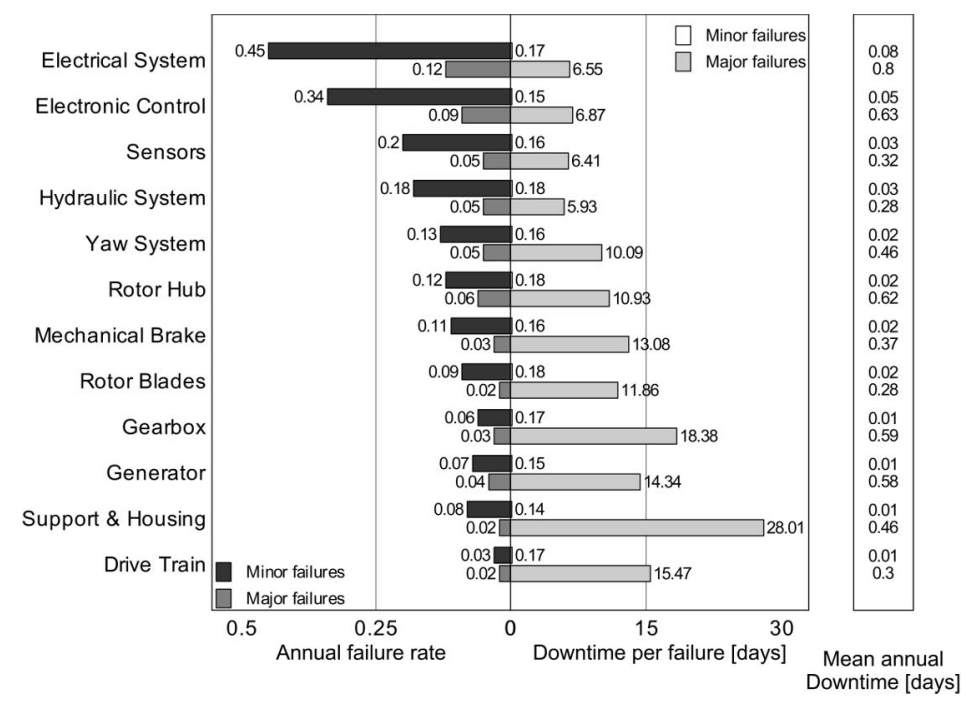

Fig. 1. Reliability Characteristics of Sub-Assemblies [4]

The tripod structure is expected to be the future of the offshore wind turbine foundation structures. This is due to the trend of setting up wind farms in deeper waters where the traditional monopole foundations will not be able to fulfill the structural requirements. The major modes of failure of the tripod structure are fatigue at the main node and scouring at the support where the tripod is connected to the sea bed. A frequency based damage detection 
technique for fatigue induced cracks has been proposed in [5]. Similar methodology may also be used for the detection and localization of the deterioration of the support condition due to scouring. The method quantifies the change in the frequency response function (FRF) of the healthy structure and deteriorated one using the root mean square difference (RMSD). But any other tool may also be used in order to monitor the change in the FRF. The Fig. 2 shows the placement of the fiber Bragg grating (FBG) strain sensors on the scaled tripod used for the validation of the methodology.

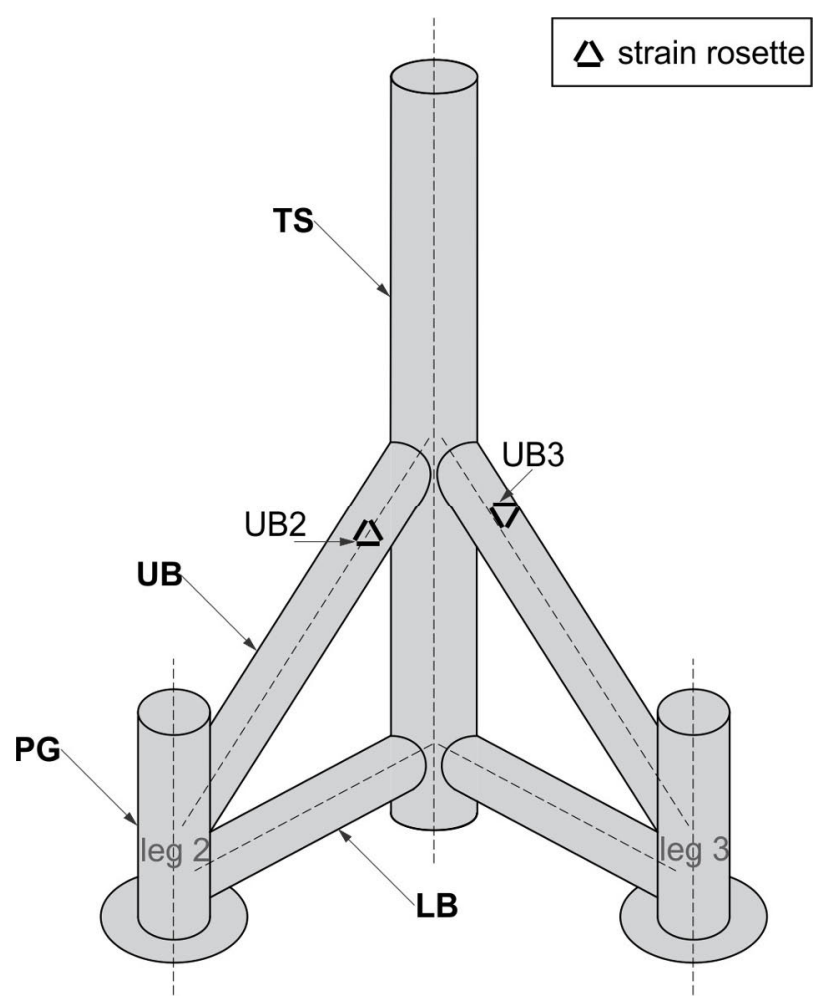

Fig. 2. Nomenclature of the components of the scaled tripod [5]; UB-Upper Brace, LB- Lower Brace, TS- Tower Support, PG- Pile Guide and UB2 and UB3 refer to the strain rosettes on leg 2 and leg 3 respectively

The FBG sensors were used due to their corrosion resistance and capability of working underwater in harsh environments. The working principle and the details about FBG sensors may be found in [6].

The strains were measured under random hammer excitation (output only) in order to simulate real conditions where the excitation of the support structure due to waves cannot be measured. The legs of the tripod were secured to the anti-shaker table with bolts and the deterioration was simulated by removing the support of one leg. The experimental validation of the methodology was limited to the extreme case while the efficacy of the method for intermediate conditions was validated using the finite element model. 
The

FRF for Strain rosette TS1

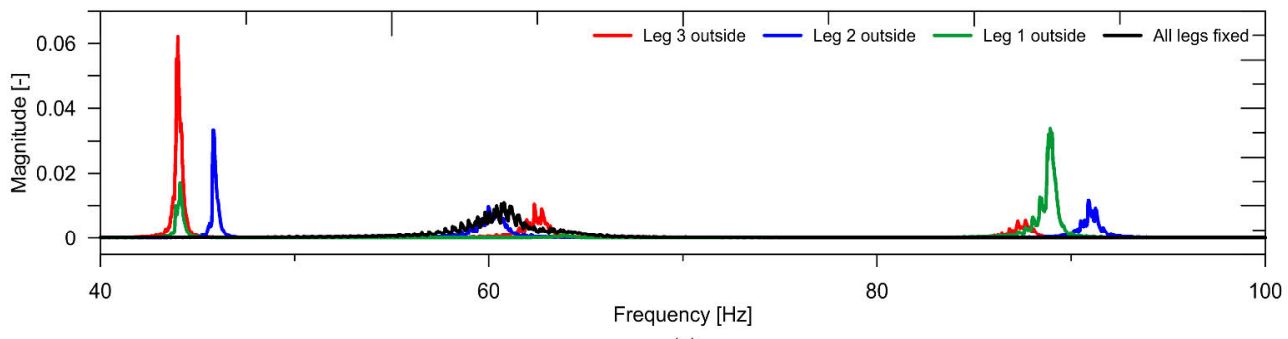

(a)

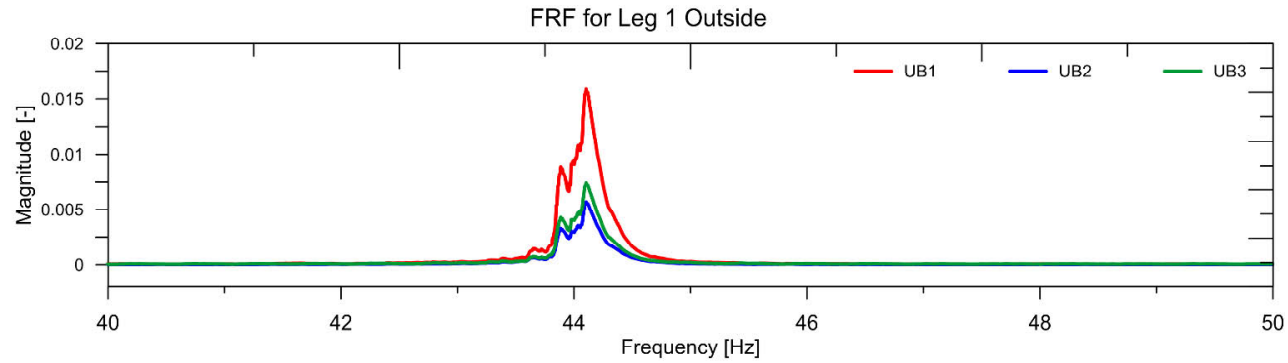

(b)

Fig. 3 (a) shows the experimental results for the four cases namely all legs bolted and each of the leg unsupported. As can be seen, the removal of the support leads to the new peaks in the FRF spectrum. If the RMSD value of the peaks is higher than a certain threshold determined based on engineering judgement the damage is said to be detected. The peaks for the damaged condition are at the same frequencies owing to the symmetry of the structure. By analyzing the new peaks in the FRF, the damage can be further localized.

FRF for Strain rosette TS1

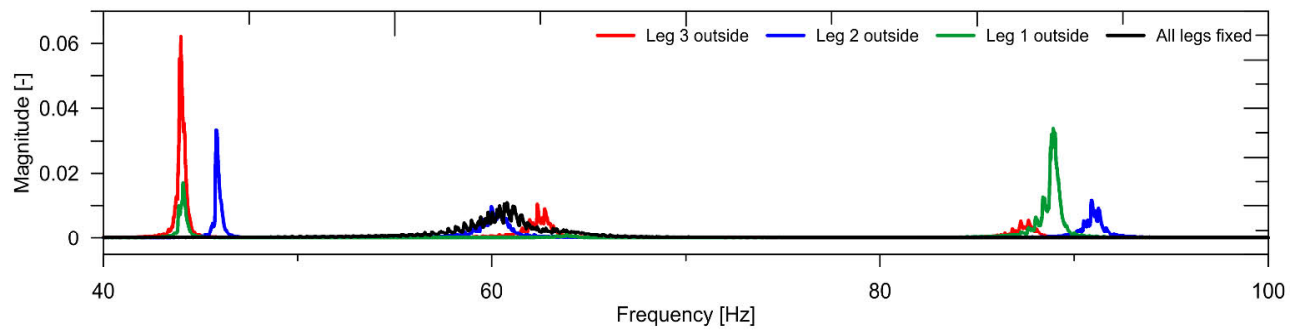

(a)

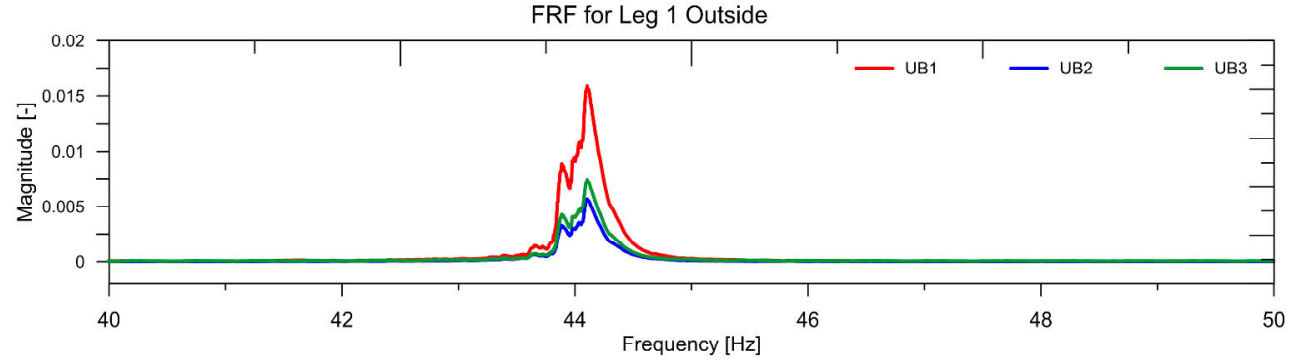

(b)

Fig. 3. FRF Spectrum for: a) each of the scenarios healthy and each leg support removed, b) each sensor on UB for the scenario Leg1 outside 
As can be seen in

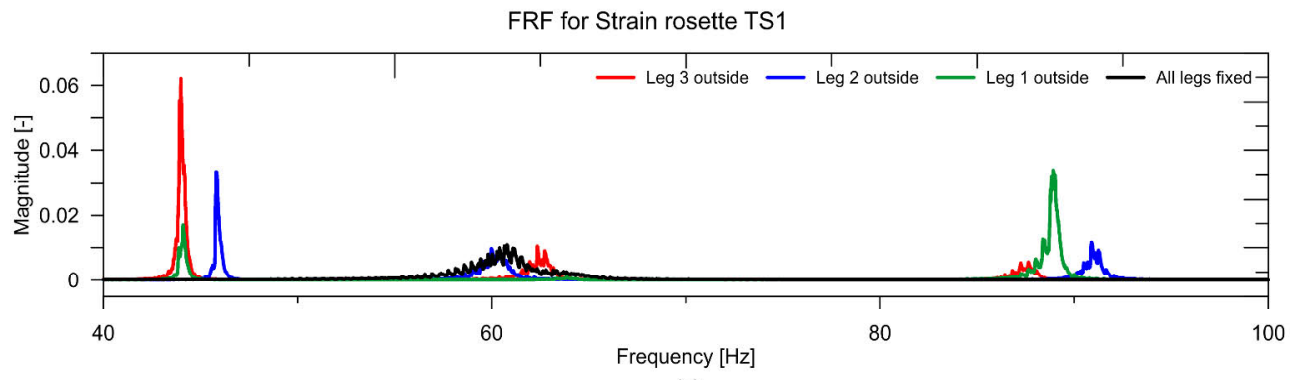

(a)

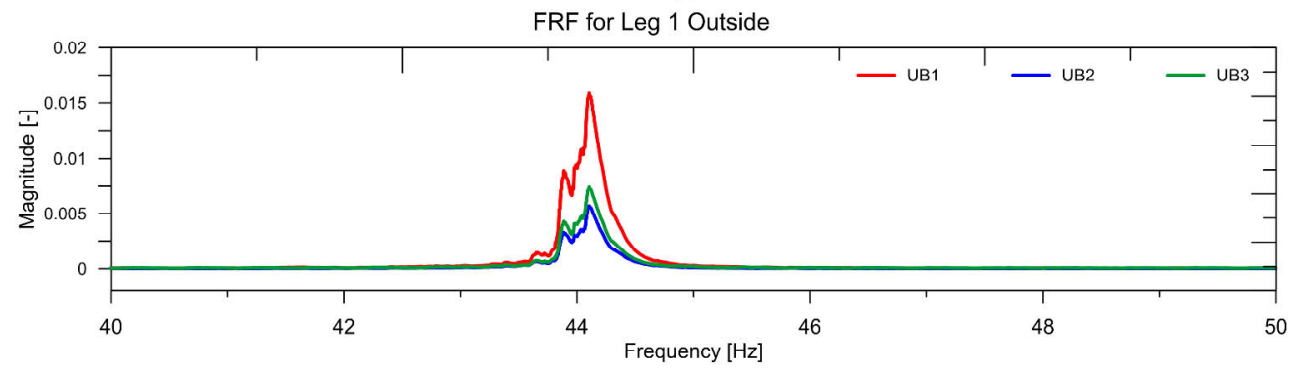

(b)

Fig. 3 (b), the peak related to the deteriorated support is highest. Although this might seem a trivial damage localization criterion, it is only valid for the vibration modes where the UB of the tripod has significant deflection. Thus a validated FE model is necessary for supporting the damage localization process. Although a development of a validated FE model is time consuming and difficult, the present practice in design of the support structures the entire wind farm has only a few standardized designs of the support based on the sea bed depth and condition. Hence, the proposed methodology is still cost effective.

\section{Composite Structures}

In this section selected non-destructive testing method for assessment of composite structures are presented. Composite structures during exploitation are exposed to mechanical damage (delamination) and physico-chemical degradation (moisture, thermal degradation, aggressive agents). Therefore, there is a need of development of assessment methods that are able to detect mentioned typed of material degradation.

\subsection{Active thermography}

First method was based on active thermography. In this approach pulse excitation based on halogen lamps $(2 \times 150 \mathrm{~W}, 7 \mathrm{~s}$ heating time) was utilized. Temperature increase due to heating was equal to $30^{\circ} \mathrm{C}$. Sensing process was performed using FLIR SC5600 infrared camera. Experimental stand was presented in Fig. 4. Research was conducted for GFRP panel with dimensions $500 \mathrm{~mm} \times 500 \mathrm{~mm} \times 1.5 \mathrm{~mm}$. In this panel $20 \mathrm{~mm} \times 20 \mathrm{~mm}$ teflon insert was located in order to simulate delamination. Result of measurement is presented on differential thermogram (Fig. 5). The location of teflon insert is clearly visible. 


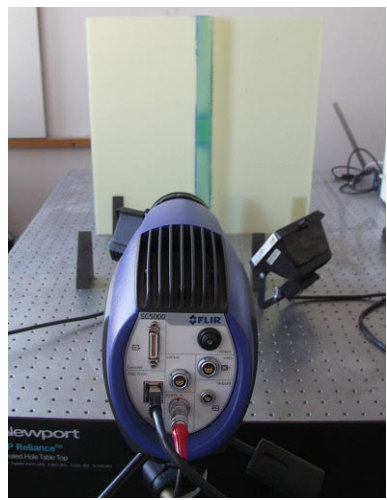

Fig. 4. Active thermography stand

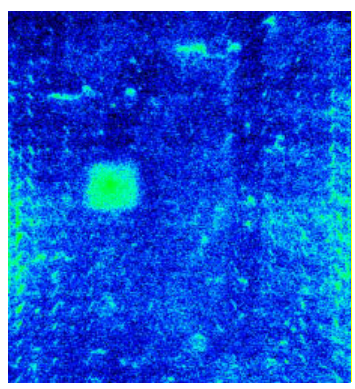

Fig. 5. Thermogram for GFRP sample with teflon insert $(20 \times 20 \mathrm{~mm})$

\subsection{Scanning Laser Doppler Vibrometry SLDV}

In this section results of experimental investigations based on Polytec PSV-400 Scanning Laser Doppler Vibrometer (SLDV) are presented. In the conducted research only one scanning laser head was utilized (measurements along laser beam approximating out-ofplane velocities). Measurements were performed for dense mesh of points spanned over the area of investigated composite panels.

A simple and widely used full wavefield signal processing method is based on root mean square (RMS) of the time domain signals. As a result energy distribution related to propagation of wave in the structure is obtained. The location and size of damage are visualized by much higher amplitudes in areas of their occurrence than in undamaged areas. Weighted root mean square (WRMS) is a modification of approach based on RMS energy. In WRMS a weight factor is added that decreases the importance of the time samples at the beginning (large wave amplitudes due to excitation) and increases the importance of the samples closer to the end of signal where waves amplitudes are small due to damping. The Weighted Root Mean Square (WRMS) can be calculated as:

$$
W R M S=\sqrt{N} \sum_{k=1}^{N} w_{k} s_{k}^{2}
$$

where coefficient $w_{k}$ is defined as follows: $w_{k}=k^{m} \quad m>0$. In the case of $w_{k}=k^{0}$ we obtain classical RMS. For case of $w_{k}=k^{1}$ linear amplification of signal amplitude with time will be obtained. Utilization of $w_{k}=k^{2}$ will give square function of amplification with time. Weighting factor $w_{k}=k^{1.8}$ was utilized in this research. 
In this research three CFRP panel with planar dimensions $500 \mathrm{~mm} \times 500 \mathrm{~mm} \times 3.5 \mathrm{~mm}$ and $5 \mathrm{~mm}$ were used. In these panels teflon inserts were located in order to simulate delamination. Three sizes of teflon inserts were investigated: $10 \times 10 \mathrm{~mm}, 15 \times 15 \mathrm{~mm}$ and $20 \times 20 \mathrm{~mm}$. Each panel has one simulated delamination. Results in the form of WRMS energy maps for investigated delamination sizes are presented in Fig 6. Strong energy concentration in the delaminated areas can be observed. These maps show location, shape and size of delaminations.

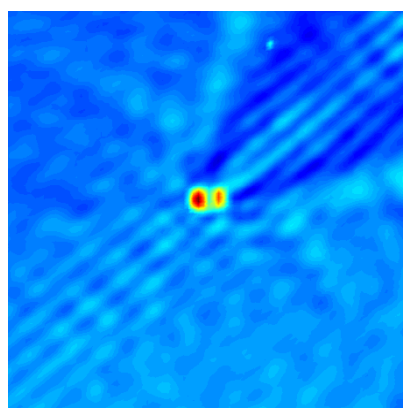

a)

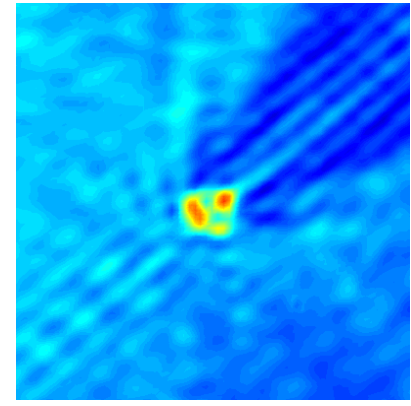

b)

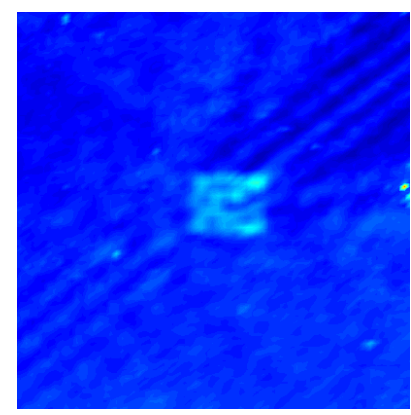

c)

Fig. 6. WRMS damage map for CFRP sample with teflon insert: a) $10 \times 10 \mathrm{~mm}$, b) $15 \times 15 \mathrm{~mm}$, c) $20 \times 20 \mathrm{~mm}$; excitation frequency $100 \mathrm{kHz}$

The next investigated case was aerospace CFRP panel with omega stiffeners. Panel consists of two CFRP laminates bonded together (one laminate includes formed stiffeners). In this panel circular delamination simulated by teflon insert with diameter $30 \mathrm{~mm}$ was located (Fig. 7). The result in the form of WRMS energy map for excitation frequency $50 \mathrm{kHz}$ was presented in Fig. 8. In the WRMS energy map stiffeners locations can be clearly observed. Moreover, location of delamination with diameter $30 \mathrm{~mm}$ can be also noticed.

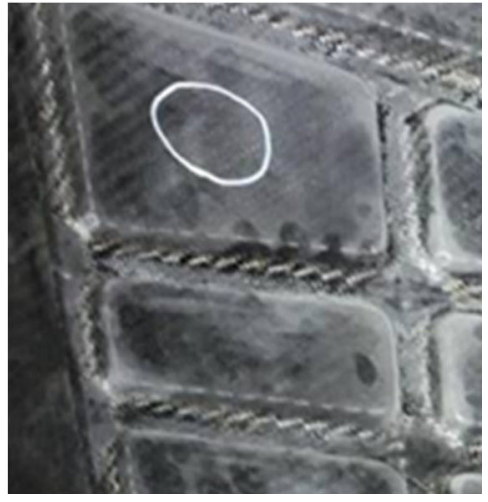

Fig. 7. CFRP stiffened aerospace panel with teflon insert (diameter $30 \mathrm{~mm}$ )

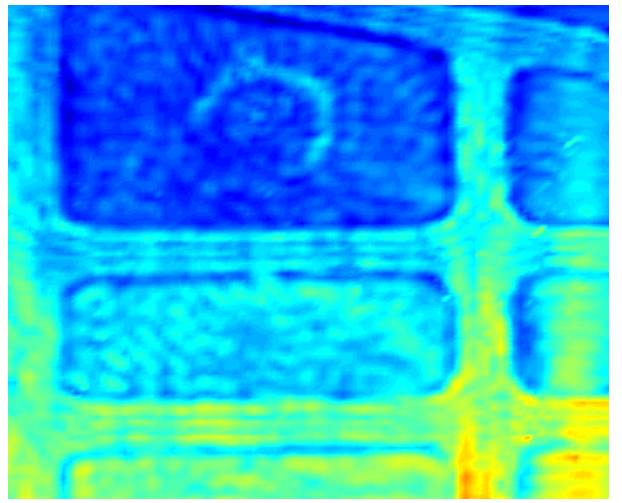

Fig. 8. WRMS damage map for CFRP stiffened aerospace panel with teflon insert; excitation frequency $50 \mathrm{kHz}$

\subsection{Laser Induced Fluorescence LIF}

In this section research was focus on a spectroscopic method that can be applied for thermal degradation detection in composite elements. In this technique spatial distribution of fluorescence intensity can be monitored with CCD imaging camera equipped with bandpass optical filters. Laser Induced Fluorescence LIF spectra were recorded using laboratory 
system equipped with laser excitation sources: DPSS cw Nd:YAG $532 \mathrm{~nm}, 0.2$ - 2 W output power (Spectra Physics) and pulsed Nd:YAG $532 \mathrm{~nm}, 6 \mathrm{~ns}$ pulse diration (Briliant B, Quantel). The $532 \mathrm{~nm}$ excitation was chosen based on previous research. Excitation at this wavelength showed maximal sensitivity to surface state condition. Fluorescence detection system was based on $0.3 \mathrm{~m}$ Czerny-Turner type monochromator (SR-303i, Andor) and spectroscopic ICCD camera (DH-740 Istar, Andor). In the detection path excitation laser radiation was blocked by a band pass filter (OG550, Shott). Fluorescence spectra were recorded in 10 randomly chosen points on sample in order to avoid local surface inhomogeneity. Additionally, the spatial distribution of laser induced fluorescence was recorded with digital camera equipped with cut-off bandpass filter (OG570, Shott). Thermal degradation of samples was carried out by means of heat gun producing stream of hot air 5 $\mathrm{mm}$ in diameter and temperature $450{ }^{\circ} \mathrm{C}$. The influence of time duration of heat source exposure was investigated. The research was conducted on CFRP. Four times of exposure were tested 5, 10, 20 and $30 \mathrm{~s}$ in this research. The photographs of the heat exposed area under the LIF laser illumination are presented in Fig. 9. The woven structure of the composite is clearly visible. The increase in the intensity in the visible light is also clearly observed. With increasing time of heating a clear circular shape forms indicating the overheated area can be observed.

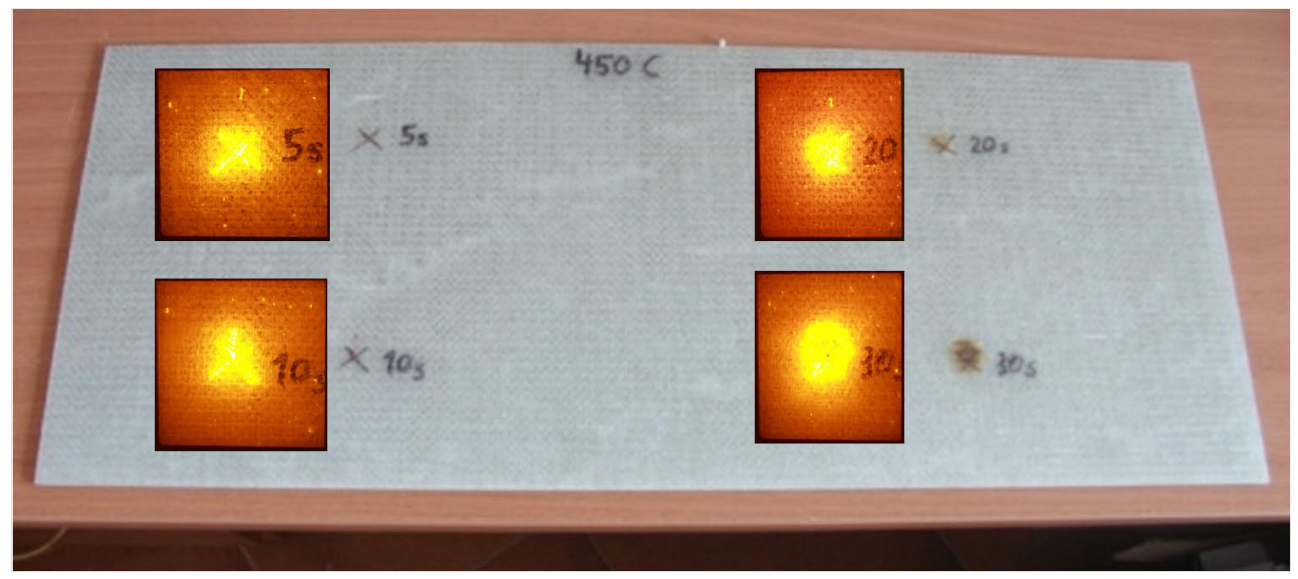

Fig. 9. Fluorescence intensity distribution registered at GFRP sample exposed to heat source at $450^{\circ} \mathrm{C}$ for $5,10,20$ and 30 seconds - pictures made with photo camera

\section{Conclusions}

In this paper different assessment method for metallic and composite parts of structures were presented. The investigation of metallic structure with the FBG sensors showed promising results. Strain tracking allowed to distinguish the damage case after analysis of the FRF response. In the case of composite elements three non-contact nondestructive testing NDT measurement techniques were discussed. Active thermography and laser vibrometry were utilized for delamination detection in composite samples. Delaminations were simulated by teflon inserts. Both method gave very good results of delamination localization. In the case of LIF method thermal degradation in samples was detected. Thermal degradation was induced by a heat gun. The LIF method also gave good results of thermal degradation localization. 
Research was partially supported by the project entitled: Non-invasive Methods for Assessment of Physicochemical and Mechanical Degradation (PBS1/B6/8/2012) granted by National Centre for Research and Development in Poland.

This research was also supported by the project titled: Development of the selection method of the offshore wind turbine support structure for polish maritime areas (PBS1/A6/8/2012, Aquilo) granted by National Center for Research and Development in Poland.

Authors would like to acknowledge the European Commission for their research grant under the project FP7-PEOPLE-2012 ITN 309395 MARE-WINT (new Materials and REliablity in offshore WINd Turbines technology).

Authors would like to give special thanks to Academic Computer Centre in Gdansk (CI TASK) for access to ABAQUS software.

The authors would like to thank Mirosław Sawczak for the research on LIF technique.

\section{References}

1. R. N. Soman, P. H. Malinowski and W. M. Ostachowicz, 2015. Bi-axial neutral axis tracking for damage detection in wind-turbine towers. Wind Energy, vol. 19, 4, pp. 639650.

2. Y. Feng, Y. Qiu, C. J. Crabtree, H. Long and P. J. Tavner, Monitoring wind turbine gearboxes, Wind Energy, vol. 16, no. 5, pp. 728-740, 2013.

3. S. Sheng, Wind turbine gearbox condition monitoring round robin study-vibration analysis, Contract, vol. 303, pp. 275-3000, 2012.

4. S. Faulstich, B. Hahn and P. J. Tavner, Wind turbine downtime and its importance for offshore deployment, Wind Energy, vol. 14, no. 3, pp. 327-337, 2011.

5. S. Opoka, R. Soman, M. Mieloszyk and W. Ostachowicz, 2016. Damage detection and localization method based on a frequency spectrum change in a scaled tripod model with strain rosettes. Marine Structures, 49, pp.163-179.

6. E. Udd and W. B. Spillman, Fiber optic sensors: an introduction for engineers and scientists. (John Wiley \& Sons, 2011). 